\title{
Intensive care for advanced HIV disease.
}

\author{
A deRuiter, A Webb, A Mindel, F Scaravilli, L Michaels, R F Miller
}

Case report ( $\operatorname{Dr} A$ deRuiter)

The patient, a 49 year old Caucasian bisexual fashion buyer, presented to his general practitioner in June 1991. After counselling he underwent HIV testing and was found to be HIV-1 antibody positive. The patient had sought HIV testing because he had noticed some reduction in his short and long term memory together with weight loss. He had experienced recurrent chest and sinus infections over the 18 months prior to HIV testing. In his past medical history Charcot Marie Tooth disease was diagnosed when he was 4 years old. In 1970 he had syphilis which was treated. He smoked ten cigarettes a day and drank modestly, at most four units of alcohol per week.

In early July 1991 the patient was referred to the London Lighthouse by his general practitioner because of his inability to cope at home, his steady physical deterioration and an obvious deterioration in his higher mental function. After assessment at the London Lighthouse he was referred to the outpatients department at The Middlesex Hospital. The patient's complaints at this time were of weight loss, generalised weakness and in particular difficulty in walking. Examination revealed seborrhoeic dermatitis on the face, oral hairy leucoplakia and oral candida. $\mathrm{He}$ was thin, with evidence of recent weight loss and although weak was able to transfer from a wheelchair to the examination couch. He was thought to have a rather flat affect on questioning. The diagnosis lay between a depressive illness or possibly HIV encephalopathy (AIDS related dementia). Zidovudine was commenced and arrangements were made for formal psychiatric assessment.

Within a week, however, the patient was admitted as an emergency to The Middlesex Hospital. He had developed a cough with purulent sputum and a fever. Associated with this there was a marked reduction in his mental function and mobility. On examination he was pyrexial: physical findings were as previously noted but in addition the patient was tachypnoeic with a respiratory rate of 20 per minute. Formal neurological assessment was difficult as the patient was rather uncooperative. He appeared very slow in answering direct questions and was disorientated in time and place. He had signs of Charcot Marie Tooth syndrome with bilateral foot drop, wasting of the anterior tibial, peroneal and quadriceps muscles together with pes cavus of the feet. There were no focal signs in the chest. The patient was so weak he was unable to get out of a chair. Investigations at this stage included a confirmatory HIV antibody test which was positive, hepatitis B serology showed him to be hepatitis B immune, syphilis serology showed him to be TPHA and FTA Abs positive and RPR negative. A full blood count revealed a haemoglobin of $11.0 \mathrm{~g} / \mathrm{dl}$, a total white count of $4.9 \times 10^{9} / 1$ (neutrophils $4.2 \times 10^{9} / 1$, lymphocytes $0.7 \times 10^{9} / 1$ ), and a normal platelet count. Urea and electrolytes and liver function tests were normal. A CD4 cell count was $70 / \mathrm{mm}^{3}$ (normal $350-2200 / \mathrm{mm}^{3}$ ). Serological tests for toxoplasmosis and for cryptococcal antigen were both negative and culture of blood and urine was negative. The chest radiograph showed patchy shadowing in both lung bases and arterial blood gases, taken with the patient breathing room air, revealed a $\mathrm{PO}_{2}$ of $9.9 \mathrm{kPa}$ and $\mathrm{PCO}_{2}$ of $4.4 \mathrm{kPa}$.

Over the next 24 hours the patient became increasingly disorientated and confused. He was given amoxycillin $500 \mathrm{mg}$ three times daily for his chest infection, ketoconazole $200 \mathrm{mg}$ once daily for his oral candidiasis and zidovudine was continued. A sample of sputum induced by inhalation of hypertonic saline was negative for both Pneumocystis carinii and acid and alcohol fast bacilli (AAFB). The patient became increasingly pyrexial and more hypoxaemic; because of a clinical suspicion of pneumocystis pneumonia intravenous high dose co-trimoxazole was commenced. On the third day of the hospital admission fibreoptic bronchoscopy was performed. The bronchoscopic appearances were of marked pharyngeal candida. In addition mucus was seen in the left bronchial tree, no endobronchial lesions were seen. Bronchoalveolar lavage was performed and this fluid was negative for $P$ carinii, AAFB and cytomegalovirus. In view of the patient's increasing disorientation cerebral CT was performed. This showed evidence of mild cortical atrophy but was otherwise unremarkable. A lumbar puncture was performed and the cerebral spinal fluid was clear, no white cells were seen and on Gram stain no organisms were detected. The India ink stain was also negative as was a test for cryptococcal antigen. The CSF protein was $0.52 \mathrm{~g} / 1$ but the CSF glucose level was a little reduced at $2.5 \mathrm{mmol} / 1$ (with a simultaneous plasma glucose of $5.2 \mathrm{mmol} / \mathrm{l}$; CSF syphilis serology was negative. By the end of day four of the patient's admission he had become rather more hypoxaemic with a $\mathrm{PO}_{2}$ of $7 \cdot 2$ breathing room air and the chest radiograph (fig 1 ) had 
Fig 1 Chest radiograph taken on day four of admission, showing bilateral patchy shadowing in both mid and lower zones.

\section{Fig 2 Chest radiograph taken after endotracheal intubation. A left sided intercostal chest drain has been inserted to trea: a left pneumothorax.}
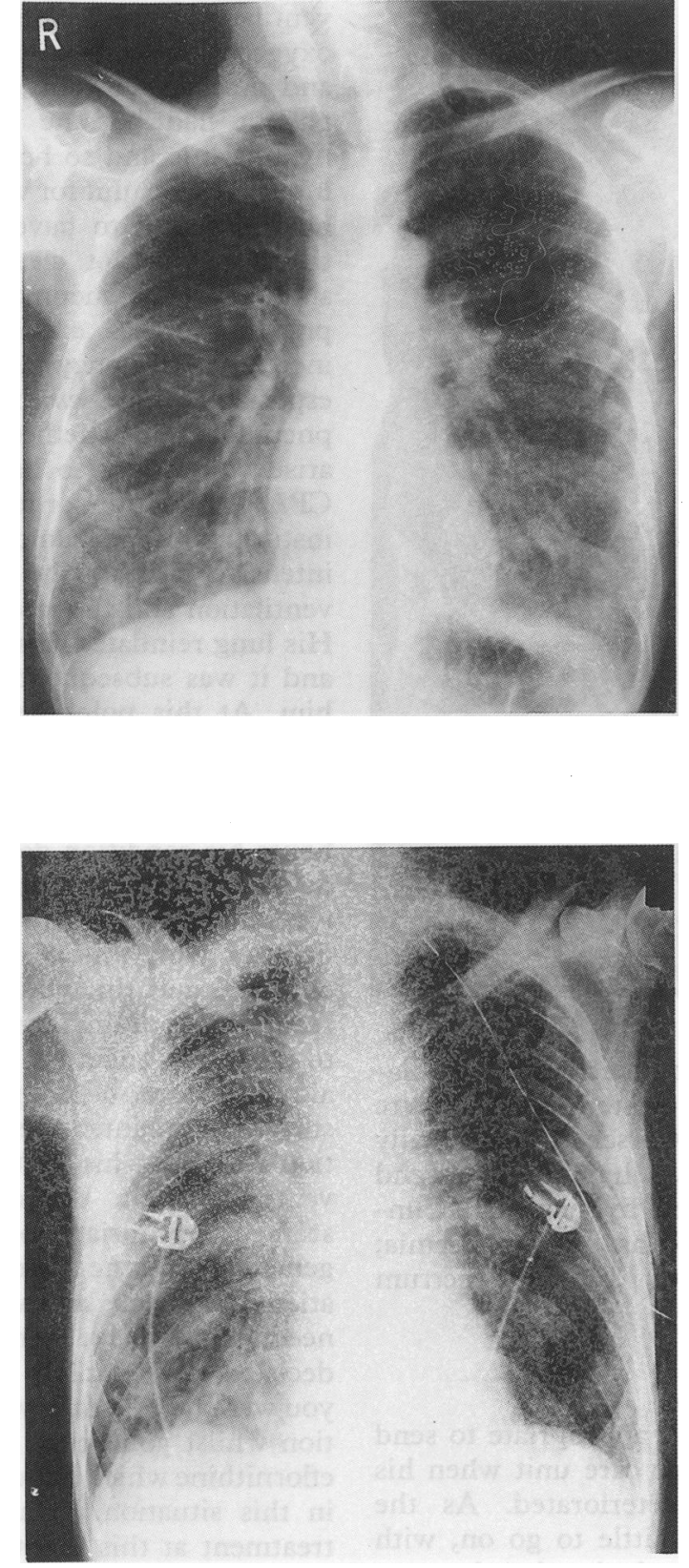

deteriorated further. Pulse megadose methylprednisolone was added at this stage because despite the negative bronchoalveolar lavage pneumocystis pneumonia was still suspected. Over the next 48 hours the patient remained confused and hypoxaemic Pseudomonas aeruginosa and Staphylococcus aureus were isolated from the induced sputum sample and bronchoalveolar lavage fluid. Methylprednisolone was discontinued and flucloxacillin was added to his antibiotic regime. Despite these manoeuvres the patient continued to deteriorate and by day seven of his admission his $\mathrm{PO}_{2}$ was only $6.0 \mathrm{kPa}$ whilst breathing $60 \%$ oxygen via a tight fitting face mask. At this point continuous positive airways pressure (CPAP) ventilation via a face mask was commenced. The patient tolerated this very well, his dyspnoea improved as did his arterial blood gases and for 48 hours his condition appeared to improve clinically.

On the eighth day of admission he deteriorated acutely, becoming tachycardic with a pulse rate of 150 per minute and tachypnoeic with a respiratory rate of 42 per minute. He became increasingly exhausted and cyanosed and was transferred to the intensive care unit. On arrival in the intensive care unit he was intubated and mechanically ventilated. A chest radiograph taken immediately after mechanical ventilation was commenced showed he had a large left pneumothorax. An intercostal chest drain was inserted. Following ventilation and insertion of the chest drain the left lung re-expanded and his condition rapidly improved (fig 2). Within 36 hours of his transfer to the intensive care unit he was extubated and was able to return to the ward with a chest drain in place.

He then remained on the general ward for the next three days. His condition was initially stable but subsequently deteriorated, with increasing exhaustion and worsening hypoxaemia. On day 12 of his admission his $\mathrm{PO}_{2}$ was only $8.5 \mathrm{kPa}$ whilst breathing $80 \%$ oxygen through a face mask. His chest radiograph at this stage showed worsening interstitial shadowing and alveolar consolidation. At this point he was transferred back to the intensive care unit. He subsequently developed a second right-sided pneumothorax which was treated with an intercostal chest drain (fig 3). Throughout this time his co-trimoxazole had been continued and at this stage intravenous pentamidine was added in addition to the cotrimoxazole. The two drugs were overlapped 48 hours and then the co-trimoxazole was stopped. The patient continued to deteriorate despite this manoeuvre and so salvage therapy with eflornithine was commenced. The dose of eflornithine had to be reduced as the patient had developed thrombocytopenia which persisted despite transfusion of platelets.

Both chest drains bubbled continuously, suggesting there was continuing air leak and also the possibility of a bronchopleural fistula. Suction was applied to both chest drains and with this the lungs remained inflated. Because of deteriorating blood gases the patient 
Fig 4 Emphysematous bullae (arrows) seen at the apex and on the medial surface of the right lung. The pale tissue at the bottom left of the picture is a piece of parietal pericardium.

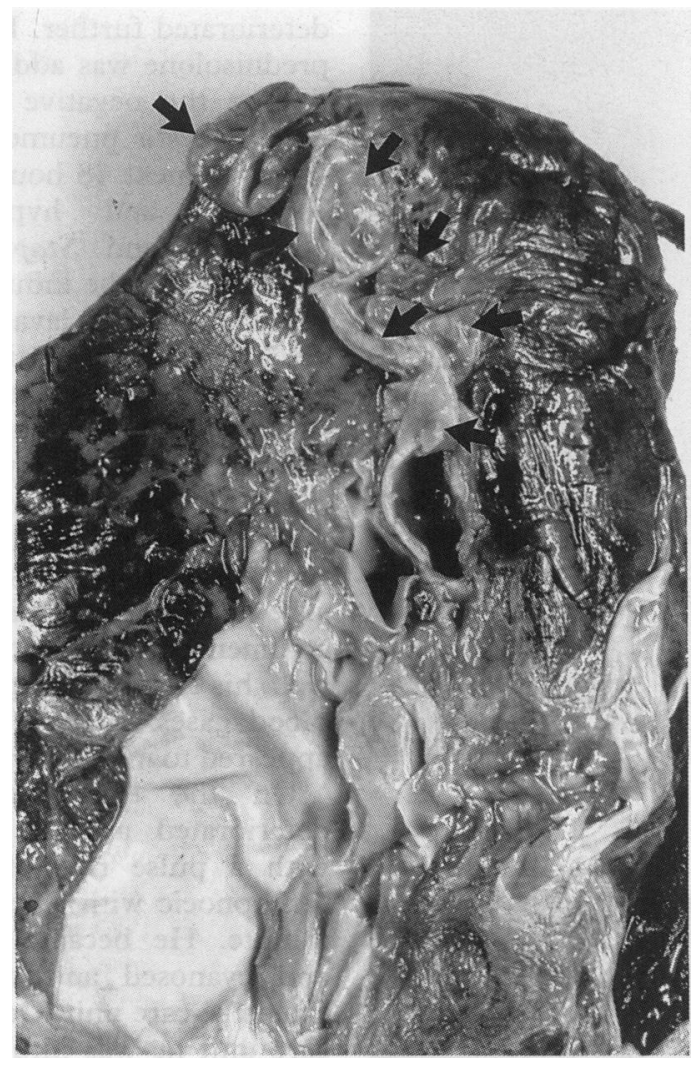

required reintubation and mechanical ventilation was reinstituted. Despite this manoeuvre the patient's condition worsened. Terminally he became pyrexial, hypotensive and tachycardic with marked hypoxaemia. Clinically he was thought to have a septicaemia; despite treatment with broad spectrum antibiotics he died.

\section{Discussion ( $\operatorname{Dr} A \mathrm{Webb})$}

I am sure it was entirely appropriate to send this man to the intensive care unit when his respiratory condition deteriorated. As the referring team you had little to go on, with respect to his long term outlook, other than you knew he had been deteriorating mentally prior to his admission to hospital. A trial of CPAP



Fig 5 Emphysematous alveoli in centrilobular distribution. There are numerous macrophages containing carbon pigment (arrows). ( $\times 10$, haematoxylin and eosin.) ventilation, used in an attempt to improve oxygenation without recourse to intubation and mechanical ventilation, had failed. ${ }^{1}$ The patient had become increasingly tired and hypoxaemic and so he was transferred to the intensive care unit for ventilation. At this point he was found to have sustained a pneumof thorax. It is not clear whether this was ब spontaneous pneumothorax (spontaneous. pneumothoraces are known to occur with increased frequency in HIV positive patient especially those with Pneumocystis carini pneumonia), or whether the pneumothorax ha⿳亠口冋. arisen secondary to barotrauma, either from CPAP ventilation, or following intubation and institution of mechanical ventilation. ${ }^{12}$ On the intensive care unit he rapidly improved wit ventilation and the insertion of a chest drairy His lung reinflated, his oxygenation improved and it was subsequently possible to extubate him. At this point he then returned to the general ward, spontaneously breathing, con? scious and alert receiving supplemental oxyger. via a face mask. Unfortunately over the next 4 hours his condition deteriorated again and $s$ he returned to the intensive care unit. He was reventilated and there were no technical difo ficulties with this. A second pneumothora occurred and this was readily treated with of second chest drain. I am sure it was appropriate to treat him again on the intensive care unit although in retrospect perhaps he should have stayed on the intensive care unit after extubation following his first period of mechanica ventilation. On the other hand though seemed appropriate to send him back to the general ward as he was maintaining his oxygen? ation adequately and was really no longer if need of intensive care at that point. The decision to reventilate I am sure was right as you were buying time with mechanical ventili tion whilst you began salvage treatment with eflornithine which has up to a $60 \%$ success rate. in this situation. ${ }^{3}$ You were also modifyin treatment at this point by beginning specific therapy for potential bacterial co-pathogen? Terminally his condition deteriorated very rapidly. I think he died from overwhelming sepsis.

\section{Dr A Mindel}

There are several aspects of this case which aft of interest, these include the Charcot Mard Tooth disease and in particular whether this had any connection with the patient's subsequent clinical course, the HIV related neurological problems, his chest problem, the coincident haematological abnormalities and finally the ethics of ventilation in patients wi advanced HIV disease.

Charcot Marie Tooth is an autosoma dominant disorder which is also known peroneal muscular atrophy. There is coB siderable overlap with the other genetic neuropathies and there may be some overle with the neurological ataxias such as Friect reich's ataxia. The illness probably had little bearing on this patient's HIV infection and vice versa, although when he originally presented with an unsteady gait and weakness some of the 
symptomatology might have been attributable to his Charcot Marie Tooth disease.

Cerebral CT showed evidence of cortical atrophy, in keeping with a diagnosis of HIV encephalopathy. Fever and hypoxaemia probably exacerbated the patient's underlying neurological condition.

At presentation the clinical and laboratory findings were very suggestive of Pneumocystis carinii pneumonia. If indeed this was pneumocystis pneumonia it is likely that it was nodular or granulomatous Pneumocystis carinii infection. It is possible that some other organisms such as atypical mycobacteria, cytomegalovirus or fungi was a complicating infection. The growth of Staphylococcus aureus and Pseudomonas aeruginosa from the bronchoalveolar lavage fluid suggests that these infections may have contributed to this man's illness and it is of interest that he did not receive any antibiotics which would have been affective against pseudomonas infection until a considerable time after his admission. One other possible diagnosis needs to be considered and this is pulmonary Kaposi's sarcoma. Against this was the observation that there were no cutaneous or oral cavity lesions of Kaposi's sarcoma and no endobronchial lesions were seen at bronchoscopy. However, the chest radiographic appearances would be compatible with the diagnosis.

This man's illness was undoubtedly complicated by the early development of a pneumothorax, perhaps related to CPAP ventilation. The rapid resolution of his symptoms of dyspnoea and tachypnoea and improvement in arterial blood gases following subsequent intercostal chest drainage and endotracheal intubation and mechanical ventilation suggests that part of the problem may have been due to the patient's exhaustion. Following his return to the ward he clearly made reasonable progress only to deteriorate rapidly two days later. The marked increase in pulmonary shadowing seen on the chest radiographs could have been due to rapidly progressive pneumocystis pneumonia, bacterial or fungal infection or to fluid accumulation. The possibility that cardiac

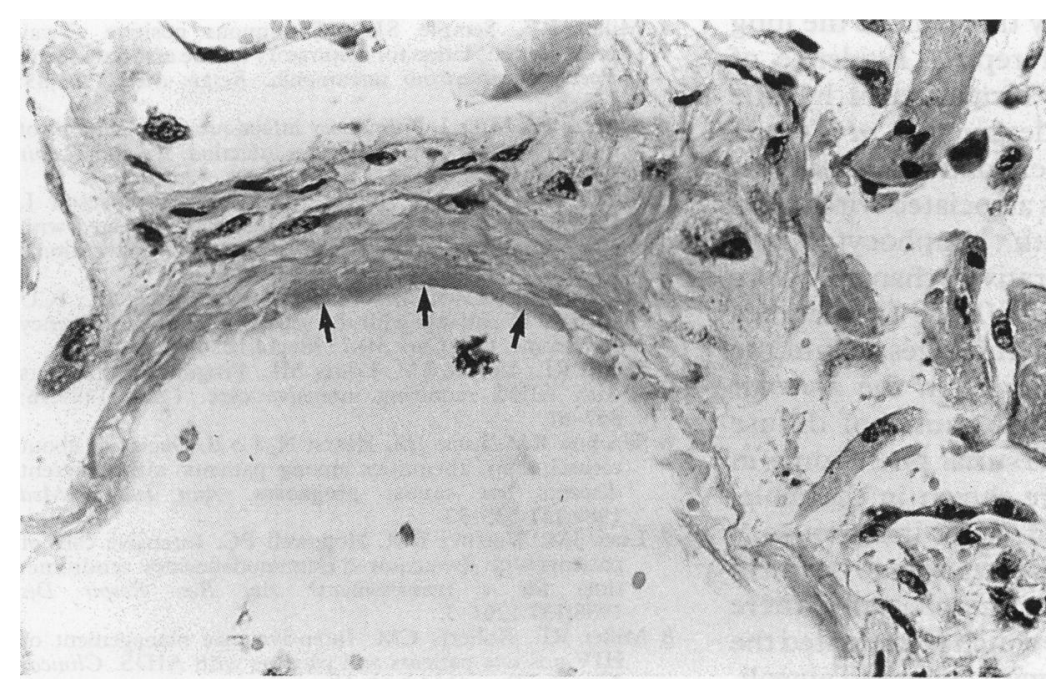

Fig 6 Loss of alveolar lining with replacement by fibrinous exudate characteristic of hyaline membrane (arrows). ( $\times 40$, haematoxylin and eosin.) failure had developed at this point needs to be considered as does the possibility of the adult respiratory distress syndrome.

There are a variety of causes for macrocytic anaemia in patients who are HIV antibody positive. The majority of patients seen in the outpatients will have a macrocytosis due to zidovudine therapy. However, other causes include rapid blood loss and subsequent increased turnover, for example from a gastrointestinal bleed, drug therapy including anticonvulsants such as phenytoin or antimicrobials such as trimethoprim, malabsorption for example due to Crohn's disease or due to ingestion of alcohol. In this patient, the macrocytosis was present prior to commencing zidovudine and there was no clear history of gastrointestinal bleeding or malabsorption and it did not appear that he drank excessive quantities of alcohol. The thrombocytopenia that developed might have been HIV related, or due to drug therapy with co-trimoxazole; systemic infection with a bacteraemia or septicaemia may also be associated with a thrombocytopenia.

Ventilation of HIV positive patients remains controversial. ${ }^{4-8}$ This procedure is probably of benefit in patients with a realistic expectation of a reasonable quality of life following the episode. ${ }^{7}$ The problem in this case was that we have very little information about the patient's mental state and quality of life prior to admission. From the limited history we have it would appear that he was reasonably well up until the time of his illness and HIV infection had not been contributing significantly to his deterioration until fairly recently. This was his first

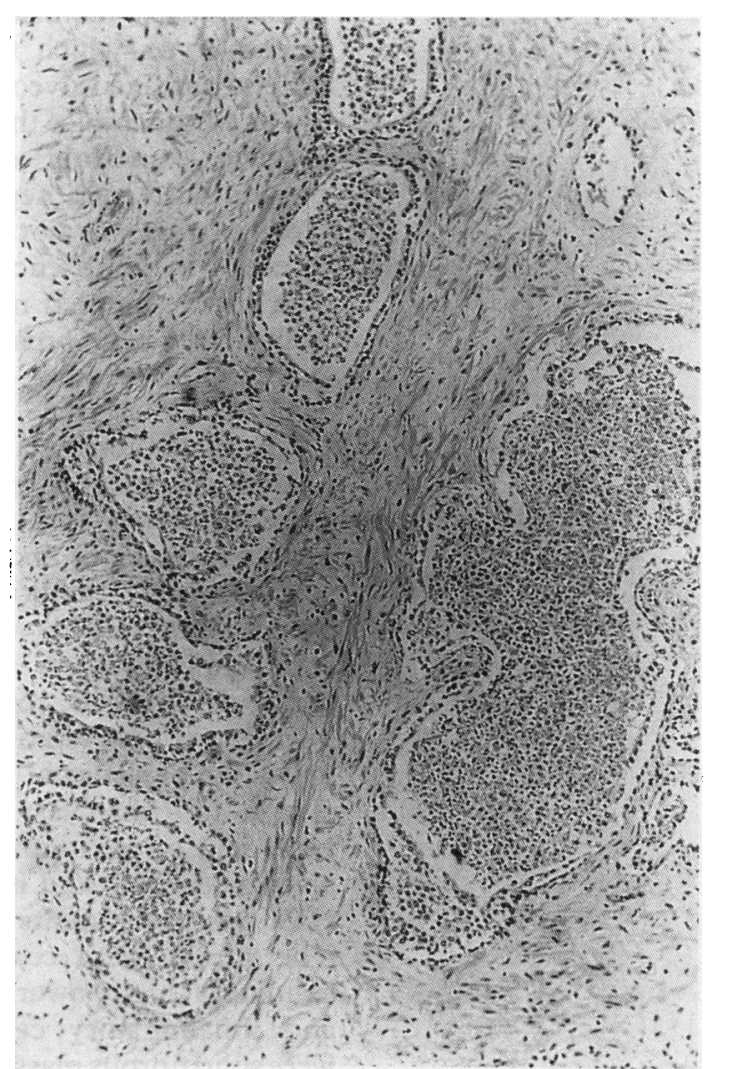

Fig 7 Acute suppurative prostatitis. Large numbers of neutrophils distend the prostatic glands. $(\times 10$, haematoxylin and eosin.) 
AIDS diagnosis and despite the very low CD4 count I believe the rationale for ventilating him was justified.

The terminal event in this man was almost certainly septicaemia. In addition I believe he had Pneumocystis carinii pneumonia, possibly complicated by either atypical mycobacterial infection, cytomegalovirus or widespread bronchopneumonia. I doubt that either the HIV encephalopathy or the Charcot Marie Tooth disease contributed significantly to his demise.

\section{Clinical diagnoses}

1 HIV encephalography

2 Pneumocystis carinii pneumonia with bacterial co-infection

3 Charcot Marie Tooth disease

4 Septicaemia as the terminal event.

\section{Pathology (Professor L Michaels)}

The body was that of a thin, wasted Caucasian man. There were clear signs of Charcot Marie Tooth disease in the legs. There were no lesions of Kaposi's sarcoma on the skin or the palate. The major pathological findings were in the lungs. Extensive apical bullae were present in both lungs (fig 4 ). When the lungs were cut open there was evidence of widespread emphysematous disease (fig 5). This was of both centri-lobular and pan-acinar distribution. Histologically the appearances of emphysema were confirmed. In addition there was an inflammatory exudate between the airspaces and the alveolar walls were broken down. Two infections were also demonstrated. Firstly, cytomegalovirus which was manifest as intracellular inclusions within Type II pneumocytes which were formed into layers around damaged alveoli. Secondly, in the bronchi and bronchioles there was an inflammatory exudate consisting of neutrophils, macrophages and Gram negative bacilli. The pathology of this was not typical of pseudomonas infection in the absence of vasculitis and infarction and resembled more that of coliform or Klebsiella pneumonia. Throughout the lungs there was no evidence of Pneumocystis carinii or AAFB.

The major abnormality throughout the lung was of destruction and repair. Evidence of destruction was seen as formation of hyaline membranes (fig 6) and destruction of Type I pneumocytes with fibrin exudation and oedema fluid formation. This was associated with interstitial inflammation with lymphocytes and neutrophils. The reparative changes were manifest as proliferation of Type II pneumocytes, the presence of macrophages within the alveoli and fibroblast activity in the alveolar walls and interstitium. The causes of diffuse alveolar damage and interstitial pneumonia in HIV positive patients are shown in the table. The pleura were coated with inflammatory exudate; at high power this was seen to be fibrin and inflammatory cells. Subpleurally there were solid fibrous masses which represented the end stage repair process with collapsed alveoli.
Table Causes of diffuse alveolar damage/interstitial pneumonia in HIV positive patients

\begin{tabular}{ll}
\hline $\begin{array}{l}\text { Infection } \\
\text { Pneumocystis carinii pneumonia } \\
\text { Gram positive cocci: }\end{array}$ & $\begin{array}{l}\text { Streptococcus pneumoniae } \\
\text { Staphylococcus aureus } \\
\text { Gram negative bacilli: }\end{array}$ \\
$\begin{array}{l}\text { Pseudomonas aeruginosa } \\
\text { Klebsiella aerogenes } \\
\text { Legionella pneumophilia } \\
\text { vytomegalovirus } \\
\text { adenovirus }\end{array}$ \\
$\begin{array}{l}\text { Toxic effects of therapy } \\
\text { Chemotherapy }\end{array}$ \\
$\begin{array}{l}\text { Radiotherapy } \\
\text { High inspired oxygen concentration, as in severe pneumonia } \\
\text { requiring mechanical ventilation. }\end{array}$ \\
\hline
\end{tabular}

In the kidneys there was haemorrhage into the pelves. In the prostate there was a florid acute prostatitis. Within the inflammatory exudate present in the prostatic glands were numerous Gram negative bacilli (fig 7). Within the adrenals there was evidence of cytomegalovirus adrenalitis. This is a common finding at post mortem in patients dying of AIDS. I don't think this would have contributed to death in this man.

\section{Neuropathology ( $D r F$ Scaravilli)}

The brain appeared normal externally and was of normal weight. Macroscopically there was diffuse calcification. Microscopically the main pathology was that of florid HIV encephalopathy. Changes seen consisted firstly of diffuse gliosis with multinucleated giant cells occurring in clumps or in a perivascular distribution, and secondly in both grey and white matter there were hyperplastic microglial cells.

\section{Pathological diagnoses}

1 HIV encephalopathy

2 Emphysema with bullae

3 Diffuse alveolar damage with cytomegalovirus colonisation

4 Gram negative bacterial lung infection

5 Gram negative prostatitis

6 Intra-renal haemorrhage

7 Cytomegalovirus adrenalitis

1 Miller RF, Semple SJG. Continuous positive airway pressure ventilation for respiratory failure associated with Pneumocystis carinii pneumonia. Respir Med 1991;85: 133-8.

2 Murray JF, Mills J. Pulmonary infectious complications of human immunodeficiency virus infection. Am Rev Respir Dis 1990;141:1582-98.

3 Smith D, Davies S, Nelson $M$, Youle $M$, Gleeson $J$ Gazzard B. Pneumocystis carinii pneumonia treated with eflornithine in AIDS patients resistant to conventional therapy. AIDS 1990;4:1019-21.

4 Schein RMH, Fischl MA, Pitchenik AE, Sprung CL. ICU survival of patients with the acquired immunodeficiency symdrome. Crit Care Med 1986;14:1026-7.

. with AIDS requiring intensive care. Chest 1989;96: w57-61.

6 Wachter RM, Luce JM, Hearst N, Lo B. Decisions abou resuscitation: Inequities among patients with different diseases but similar prognoses. Ann Intern Med 1989;111:525-32.

7 Luce JM, Wachter RM, Hopewell PC. Intensive care of patients with the acquired immunodeficiency syndrome: time for a reassessment? Am Rev Respir Di 1988;137:1261-3.

8 Miller RF, Roberts CM. Intensive care management of HIV positive patients and patients with AIDS. Clinical Intensive Care 1991;2:17-25. 